\title{
Angular-momentum-driven chaos in small clusters
}

\author{
Ersin Yurtsever* \\ Chemistry Department, Koç University, Istinye, Istanbul 80860, Turkey
}

(Received 6 January 1998)

\begin{abstract}
The effects of the rotational motion on the chaotic behavior of triatomic Lennard-Jones clusters are studied. A set of initial momentum distributions with tunable parameters is chosen to correspond to various rigid-body rotations around symmetry axes of the cluster. By smoothly varying the direction of the initial kicks given to the cluster, periodic transitions between regular and chaotic regimes are obtained. A study of initial conditions leading up to such transitions shows that the major factor that determines the extent of the chaotic behavior is the initial partitioning of the kinetic energy between the rotational and vibrational motion. From the analysis of the time evolution of various properties it is concluded that the basic role of this initial partitioning is to control the energy transfer between the kinetic and the potential energy. [S1050-2947(98)01007-5]

PACS number(s): 36.40.-c, 05.45.+b
\end{abstract}

\section{INTRODUCTION}

Classical mechanical studies of clusters present interesting problems. A clear understanding of the fundamentals of the dynamics of processes such as isomerization, fragmentation, or finding pathways leading to local or global minima requires detailed information on the potential-energy surfaces $[1,2]$. Within the adiabatic approach, the atoms move along the $(3 N-6)$-dimensional hypersurface and the optimum points along this surface determine the behavior of the system. Unfortunately, the number of optimum points along the surfaces increases almost exponentially with the size of the cluster and their identification as well as classification requires different approaches [3,4]. For example, there are over 1300 distinct minima for a cluster of 13 atoms and this number may reach $5 \times 10^{5}$ for a 19 -atom cluster. In these cases it is necessary to resort to statistical approaches rather than collecting data on characteristics of individual optimum points [5]. Then the topology of these hypersurfaces and their statistical interpretations may provide the crucial links to the dynamics on the surface [6].

On the other hand, studying small clusters even with three atoms could also pose significant challenges. The potentialenergy surface of a triatomic Lennard-Jones cluster is extremely simple with a mimimum at the equilateral triangle and three linear saddle points that are nonsuperimposable permutational isomers. However, the dynamics even along such a simple surface shows a rich variety of chaotic behavior [7-9]. The presence of chaos may be interpreted as a sign of the rapid redistribution of the vibrational energy.

The chaotic behavior of nonrotating triatomic clusters has been analyzed by Berry and co-workers in great detail $[7,8,10]$. The basic characteristics of the dynamics can be described as a function of the Hamiltonian and the topology of the surface. At low-energy values, the dynamics can be simply understood in terms of the normal modes and the cluster behaves regularly, that is, the maximum Lyapunov exponent as well as the Kolmogorov entropy is zero. As the energy increases the chaos settles in until the saddle point is

*Electronic address: eyurtsev@ku.edu.tr reached. At that point it is observed that the saddle region can cluster the close trajectories so that the degree of chaos is reduced. At larger clusters (such as five- or seven-atom clusters), this regularization of the dynamics can be observed only for shorter-range potentials and Berry has deduced that the shape of the energy surface near the saddle plays an important role. In particular, when some of the flat saddles decouple several vibrational modes, almost quasiperiodical motion can be observed.

We have also carried out extensive calculations on triatomic Lennard-Jones clusters where the effects of the initial geometry [11] and the angular momentum are studied. It was observed that whether the initial geometry corresponds to the minimum or to the saddle point, results do not really change, indicating a fast isomerization. On the other hand, the effect of the angular momentum, that the introduction of the rotational motion seemed to regularize the dynamics, was surprising. Our more detailed calculations [12] showed that indeed the nonrotating clusters displayed more chaotic motion and we concluded that the kinetic energy stored in vibrational modes played an important role in the extent of chaos.

In this work we report our calculations on rotating clusters with specifically chosen initial conditions such that periodic transitions between regular and chaotic motion can be generated. By controlling the initial kick given to the cluster we show that the tuning of the angular momentum and consequently the partitioning between the vibrational and rotational kinetic energy is the dominant factor that determines the chaotic behavior.

\section{COMPUTATIONS}

The dynamics of the cluster is followed by a four-step Runge-Kutta integration of Hamilton's equations of motion. Lyapunov exponents are computed within the tangent space formalism $[8,13]$. This approach eliminates the numerical complications arising from the exponential divergence of the close-lying trajectories. At the beginning of the integration an infinitesimal hyperellipsoid is placed at the origin of the $6 N$-dimensional phase space. The equations of motion are then written for the differences between the phase-space vector and vectors corresponding to the axes of the hyperellip- 
soid. Since these differences are small, their evolution can be written as Taylor series and the linearized form of the equations can be obtained by the first terms of the expansion. The final linear equations to be solved are

$$
\frac{d \delta x^{k}(t)}{d t}=\left.\frac{\partial F[x(t)]}{\partial x}\right|_{x=x(t)} \delta x^{k}(t) .
$$

Here $\delta x^{k}$ defines the vector with $6 N$ elements of the $k$ th axis and $F[x(t)]$ is the vector governing the time evolution of the trajectory. In our case $F[x(t)]$ holds Hamilton's equations for coordinates and momenta. Equation (1) probes the space in the vicinity of the vector $x(t)$. Since $\delta x^{k}(t)$ also tends to diverge in the case of chaotic dynamics, it has to be orthonormalized periodically. The Lyapunov exponents are calculated from

$$
\lambda_{k}=\lim _{t \rightarrow \infty} \frac{d_{k}(t)}{d_{k}\left(t_{0}\right)}
$$

where $d_{k}$ denotes the length of the $k$ th axis. Each time the vectors are orthonormalized by a Gram-Schmidt procedure, the lengths of the axes are recorded and the Lyapunov exponent spectrum is calculated from Eq. (2).

Classical trajectories are integrated up to $1.2 \mathrm{~ns}$ with time steps of 50 a.u. (approximately for $1 \mathrm{fs}$ ). The fluctuations from the average energy remain on the order of $10^{-6}$ and the sum of all 18 Lyapunov exponents is around $10^{-8}$ bits/ps. The integration of Hamilton's equations of motion can be carried out independently of the Lennard-Jones potential function parameters $\epsilon$ and $\sigma$; however, we have used the values $\epsilon=7.065 \times 10^{-21} \mathrm{~J}, \sigma=3.35 \AA$, and a mass of 12.0 proton units to compare with our previous results. The qualitative results presented here do not depend on the values of these parameters.

The initial geometry of the cluster is always chosen as the equilateral triangle that is the global minimum for the triatomic Lennard-Jones cluster. The molecule is placed on the $x y$ plane with coordinates $(0,0,0), \quad(a, 0,0)$, and $(a / 2, \sqrt{3} a / 2,0)$, where $a$ is the bond length for the dimer $\left(\sigma 2^{1 / 6}\right)$. The initial momentum components are defined in such a manner that the initial kicks correspond to the rotations around certain symmetry axes of the cluster. These kicks are controlled by two different parameters: $B$, which defines the strength, and $\theta$, which determines the direction. Of course, due to the interactions between atoms, the symmetry of the molecule and the rotations are disturbed fairly quickly. By tuning two parameters of the rotation we are able to isolate the effects of the initial values of the rotational and vibrational kinetic energy on the chaotic behavior.

The first of these rotations (I) corresponds to the rotation around the $C_{3}$ axis (Fig. 1). The momentum vectors of atoms for this rotation are defined as

$$
\begin{gathered}
\overrightarrow{P_{1}}=m B[\cos (\theta+4 \pi / 3) \vec{i}+\sin (\theta+4 \pi / 3) \vec{j}], \\
\overrightarrow{P_{2}}=m B[\cos (\theta) \vec{i}+\sin (\theta) \vec{j}], \\
\overrightarrow{P_{3}}=m B[\cos (\theta+2 \pi / 3) \vec{i}+\sin (\theta+2 \pi / 3) \vec{j}] .
\end{gathered}
$$

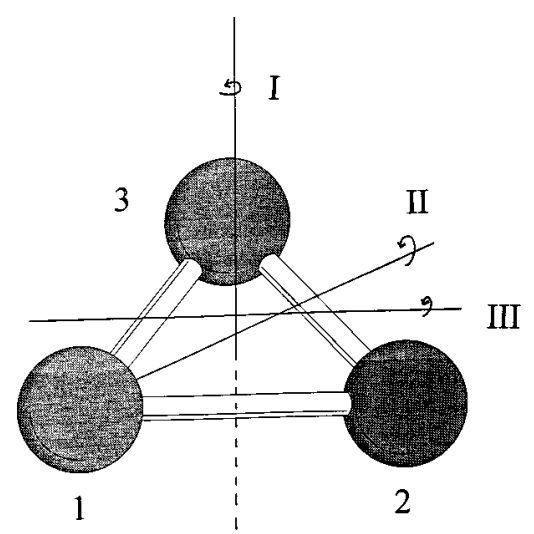

FIG. 1. Rotations I-III.

The magnitude of the kinetic energy is controlled by the parameter $B$. The angular momentum $\vec{L}$, the angular velocity $\vec{\omega}$, and the kinetic energy of the cluster $K$ can be described in terms of the parameters $B$ and $\theta$ as

$$
\begin{gathered}
\vec{L}=\frac{3 m a B}{2}\left[\frac{1}{\sqrt{3}}(\cos \theta)+\sin \theta\right] \vec{k}, \\
\vec{\omega}=\frac{1}{m} \vec{L},
\end{gathered}
$$

and

$$
K=\frac{9 m a B}{8}\left[\frac{1}{3}\left(\cos ^{2} \theta\right)+\sin ^{2} \theta+\frac{2}{\sqrt{3}}(\sin \theta) \cos \theta\right] .
$$

Since we are dealing with the nonrigid rotation, the angular velocity does not remain constant. So during the simulation we calculate the instantaneous moment of inertia tensor and the angular velocity, which allow us to partition the kinetic energy in terms of vibrational and rotational components. This analysis [14] allows one to study the kinetic-energy transfer between the vibrational and rotational motion.

The second type of motion (II) is the rotation around the bisector that connects the first atom to the midpoint of the side 2-3. The initial values of the linear momentum components, angular momentum, and the kinetic energy are

$$
\begin{gathered}
\overrightarrow{P_{1}}=0, \\
\overrightarrow{P_{2}}=m B\left[-\frac{1}{2}(\cos \theta) \vec{i}+\frac{\sqrt{3}}{2}(\cos \theta) \vec{j}+(\sin \theta) \vec{k}\right], \\
\overrightarrow{P_{3}}=-\overrightarrow{P_{2}}, \\
\vec{L}=\frac{m a B}{2}[-\sqrt{3}(\sin \theta) \vec{i}-(\sin \theta) \vec{j}],
\end{gathered}
$$

and

$$
K=\operatorname{maB}\left(\sin ^{2} \theta\right) .
$$




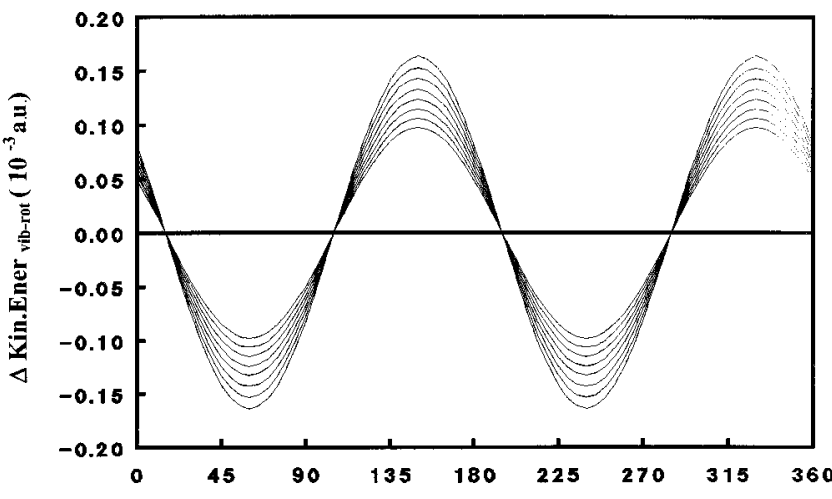

(a)

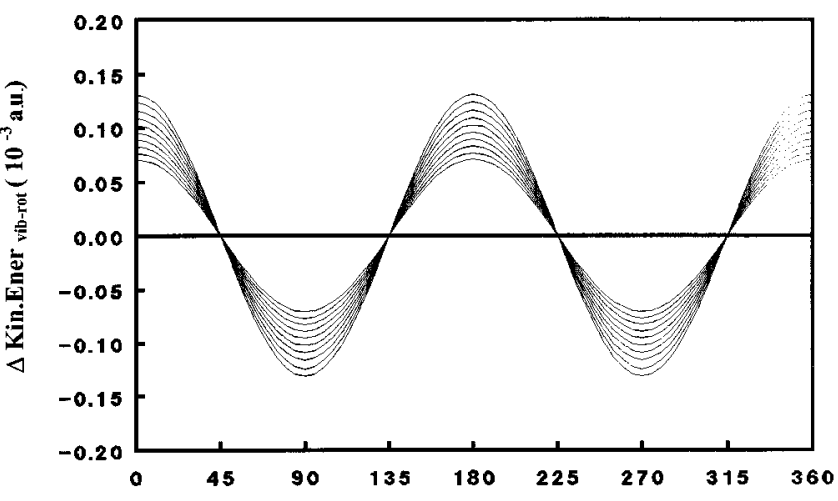

(b)

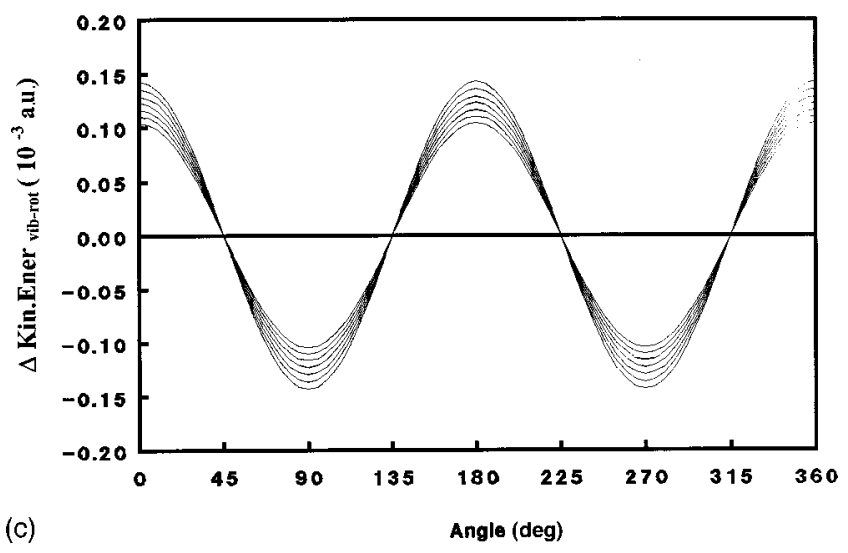

FIG. 2. Dependence of the initial value of $\Delta=E_{v i b}-E_{\text {rot }}$ on the direction parameter for $\theta$. $B$ changes from 1.20 to 1.55 for type I (a), from 1.25 to 1.70 for type II (b), and from 1.70 to 2.05 for type III (c). $\Delta$ is in a.u.

Finally, the third rotation (III) is defined as the rotation around the axis that bisects both sides 13 and 23:

$$
\begin{gathered}
\overrightarrow{P_{1}}=\frac{m B}{2}[(\cos \theta) \vec{j}+(\sin \theta) \vec{k}], \\
\overrightarrow{P_{2}}=\overrightarrow{P_{1}}, \\
\overrightarrow{P_{3}}=-\overrightarrow{P_{1}}-\overrightarrow{P_{2}}, \\
\vec{L}=-\frac{\sqrt{3} m a B}{2}(\sin \theta) \vec{i},
\end{gathered}
$$
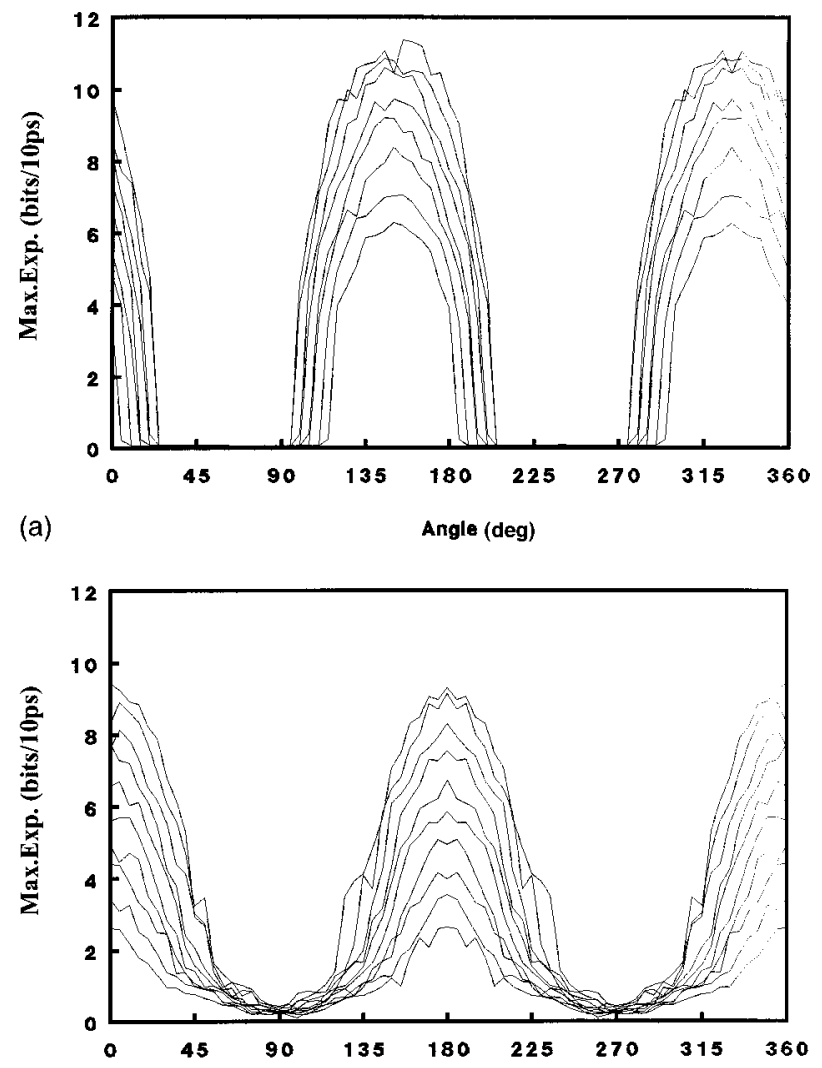

(b)

Angle (deg)

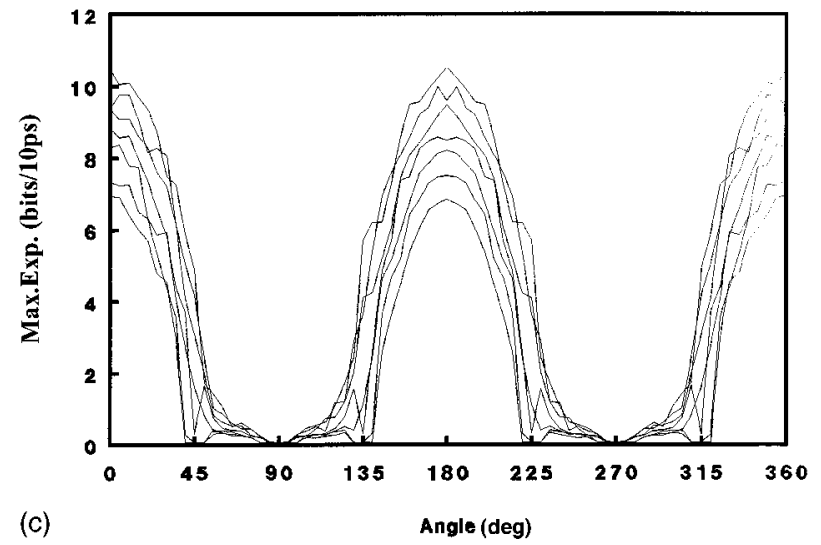

FIG. 3. Maximum Lyapunov exponents in terms of the angle $\theta$ ( $\lambda_{\max }$ is in units of bits/ps) for rotations (a) I, (b) II, and (c) III.

and

$$
K=\frac{3 m a B}{4} \sin ^{2} \theta
$$

For all rotations, the angle $\theta$ is varied between 0 and $2 \pi$ with increments of $\pi / 18$. The results for $\pi \geqslant \theta \geqslant 2 \pi$ are as symmetrical as the numerical results of a chaotic integration allow. The strength parameter $B$ is usually varied from 1.0 to 2.2 a.u., however, depending on the rotation type, different ranges are studied.

\section{RESULTS AND DISCUSSION}

The Lyapunov exponent spectra for the three types of above-defined rotations are calculated and the initial condi- 


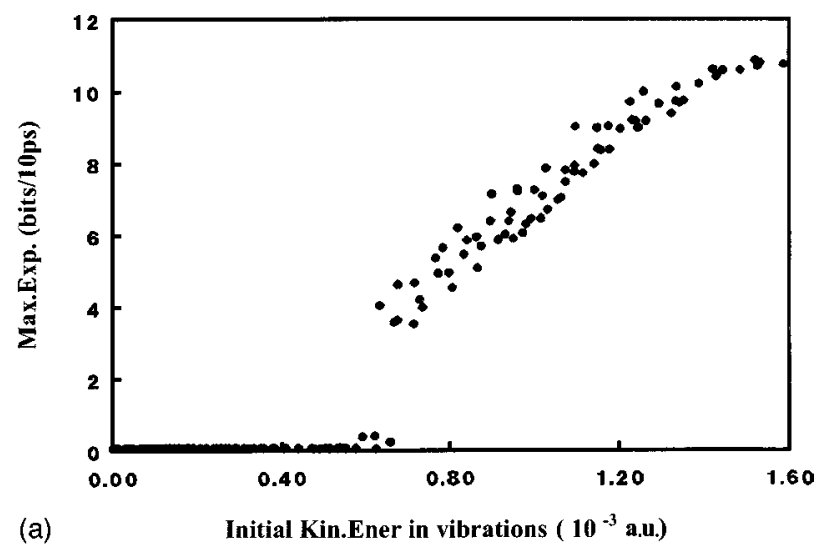

(a)
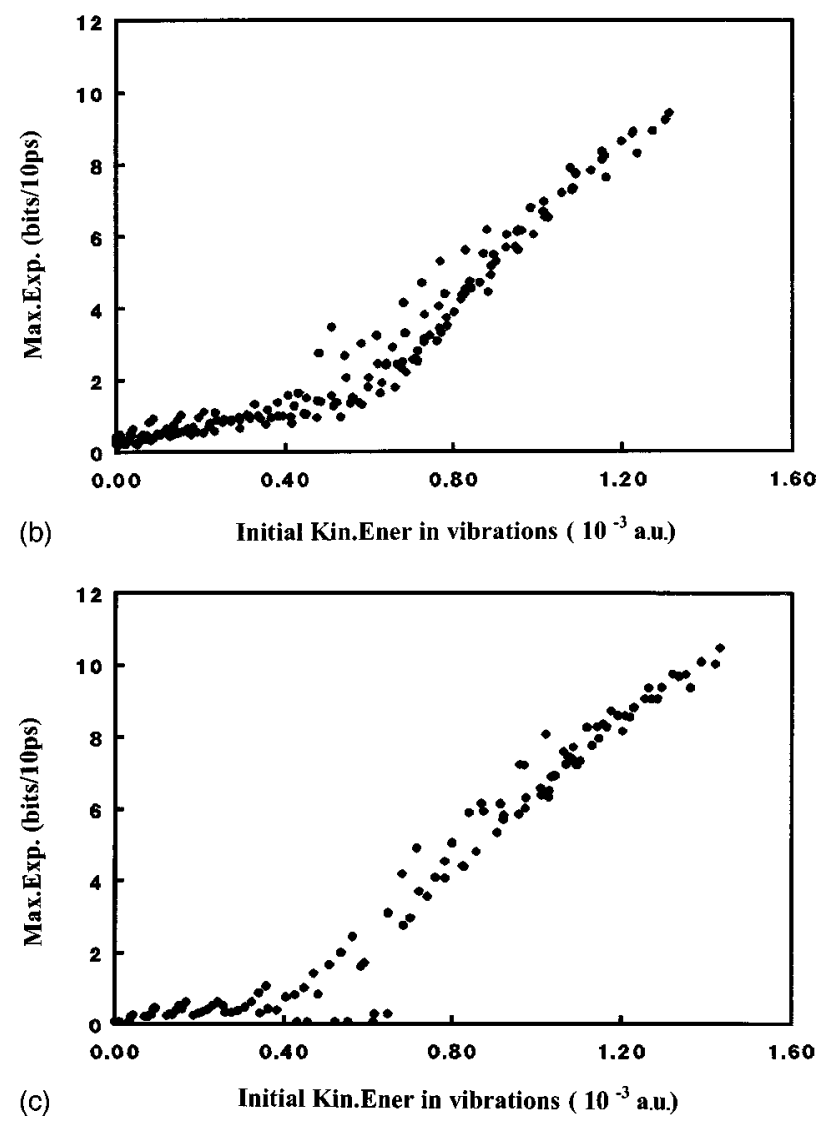

FIG. 4. Maximum Lyapunov exponents in terms of the initial kinetic energy in vibrational motion (for $10^{-4}$ a.u.) for rotations (a) I, (b) II, and (c) III.

tion dependence is studied. The parameters for this study are chosen as the strength of the kick $B$, the directional angle $\theta$, the components and the magnitude of the angular momentum, and the initial partitioning between the vibrational and rotational kinetic energies. The rotational kinetic energy is calculated from

$$
E_{r o t}(t)=\frac{1}{2} \vec{L} \cdot \vec{\omega}(t),
$$

where $\vec{\omega}$ is a time-dependent property that is obtained from

$$
\vec{\omega}(t)=I^{-1}(t) \vec{L}
$$

TABLE I. Initial conditions for the three selected trajectories. Angular momentum and energy terms are in atomic units and Lyapunov exponents are in bits/ps.

\begin{tabular}{lccc}
\hline \hline Property & Rotation I & Rotation II & Rotation III \\
\hline$\theta$ & $\pi / 2$ & $2 \pi / 3$ & $5 \pi / 6$ \\
$L_{x}$ & -10.448 & -9.048 & -5.224 \\
$L_{y}$ & -6.032 & -5.224 & -3.016 \\
$L$ & 12.064 & 10.448 & 6.032 \\
$E_{r o t}$ & $1.312 \times 10^{-4}$ & $9.387 \times 10^{-5}$ & $3.280 \times 10^{-5}$ \\
$E_{v i b}$ & 0.0 & $3.280 \times 10^{-5}$ & $9.837 \times 10^{-5}$ \\
$\Delta$ & $-1.312 \times 10^{-4}$ & $-6.56 \times 10^{-5}$ & $6.56 \times 10^{-5}$ \\
$\lambda$ & 0.4172 & 1.4301 & 7.0896 \\
\hline \hline
\end{tabular}

$I(t)$ is defined as the instantaneous tensor of inertia of the rigid body in the laboratory-oriented system of coordinates [14]. These time-dependent nonrigid-body measures also help to follow the trajectory in the phase space and determine the periodicity and transitions to chaos. Once the rotational kinetic energy is calculated, the remaining kinetic energy is stored in the vibrational motion. By modifying the strength parameter $B$ and the angle $\theta$ we generate smoothly varying functions of initial conditions. In Fig. 2 the energy partitioning between rotational and vibrational kineticenergy components $\Delta=E_{v i b}-E_{\text {rot }}$ are given as functions of the initial angle $\theta$ for various values of $B$. In rotation $I$ at $\theta=\pi / 2$ the vibrational and rotational kinetic energies are equal: The kinetic energy is transferred completely to the rotational motion at $\theta=\pi / 3$ and to the vibrational motion at $\theta=5 \pi / 6$. This behavior has a period of $\pi / 4$. Rotations II and III are similar in that optimum points are at $\pi / 4, \pi / 2$, and $\pi$. The maximum Lyapunov exponents are plotted in Fig. 3 as functions of the angle $\theta$. The maxima for the Lyapunov exponents coincide exactly with the maxima of $\Delta$. The strength parameter $B$ plays the expected role that when it is very small, the system behaves regularly as there is relatively little energy available for the kinetic energy. When $B$ increases the chaos starts to appear and for high $B$ values the integration becomes highly unstable. In Fig. 4 the maximum Lyapunov exponents are plotted as functions of the initial vibrational kinetic energy. Previously we have reported Lyapunov exponents of a large set of randomly generated initial conditions $[11,12]$ and due to the random

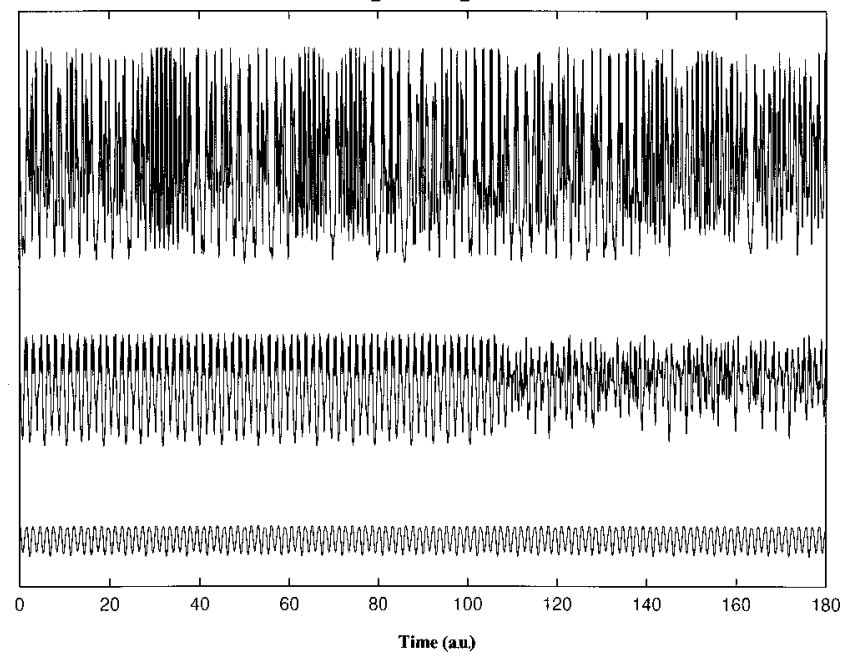

FIG. 5. Total-kinetic-energy variation in time (ps) for three trajectories with type II, $B=1.70$, and $\theta$ for $\pi / 2,2 \pi / 3$, and $5 \pi / 6$. 


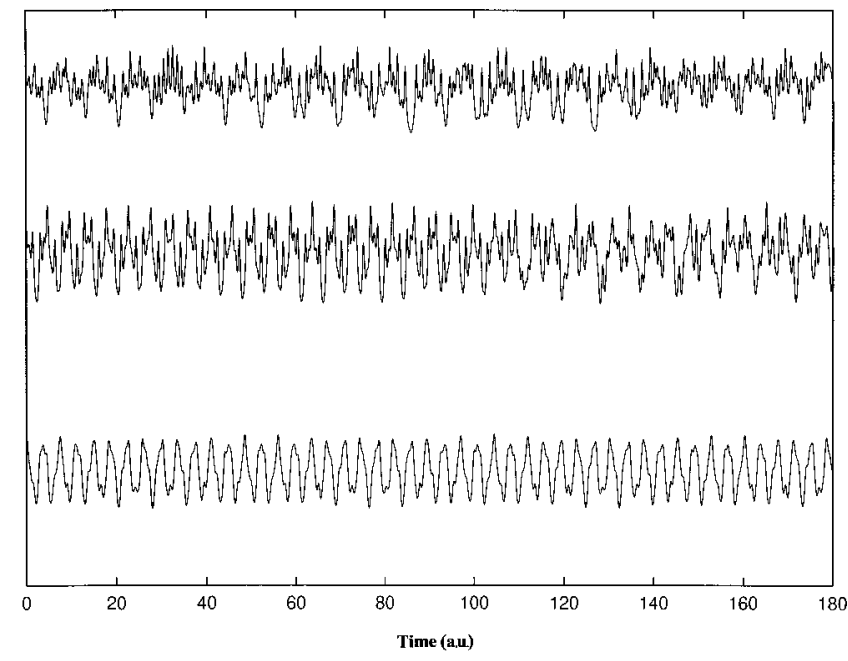

FIG. 6. Variation of the angular velocity.

nature of the set it has not been possible to isolate individual contributions to chaos from different factors. Now, by tuning the rotational motion we conclude that the initial partitioning of the rotational and vibrational kinetic energies is the major contributor to the extent of chaos. In Fig. 4 it is clearly seen that for every rotation type a threshold for the vibrational kinetic energy exists for chaos to be observed. Once this critical value is reached, $\lambda_{\max }$ linearly increases with the initial vibrational kinetic energy.

These results present an interesting analysis of the energy transfer in small clusters. For the nonrigid motion there are two distinct energy transfer channels, namely, between potential and kinetic energies and between vibrational and rotational kinetic energies. In order to study the effects of the initial conditions on these processes we study various properties as time series for three distinct cases. They are chosen such that the potential and the total energies are the same, but the kinetic energy is partitioned differently. For this purpose trajectories from rotation type II with $B=1.7$ and the angles of $\pi / 2,2 \pi / 3$, and $5 \pi / 3$ are selected. In Table I we present initial conditions and the maximum Lyapunov exponents for these trajectories. In Figs. 5-7 the total kinetic energy, the magnitude of the angular velocity, and the internal angle $\phi(312)$ are plotted as functions of time (the rotational kinetic energy depends linearly on the angular velocity and hence it shows the same behavior). The time series for each variable are shifted in arbitrary units for clarity in the figures; however, their amplitudes remain as they are calculated to allow comparisons. The first trajectory has an almost zero $\lambda_{\max }$ and all times series show a great deal of periodicity. The coupling between kinetic- and potential-energy terms is rather weak and the cluster is not able to gain sufficient energy to display chaotic behavior. The variation of

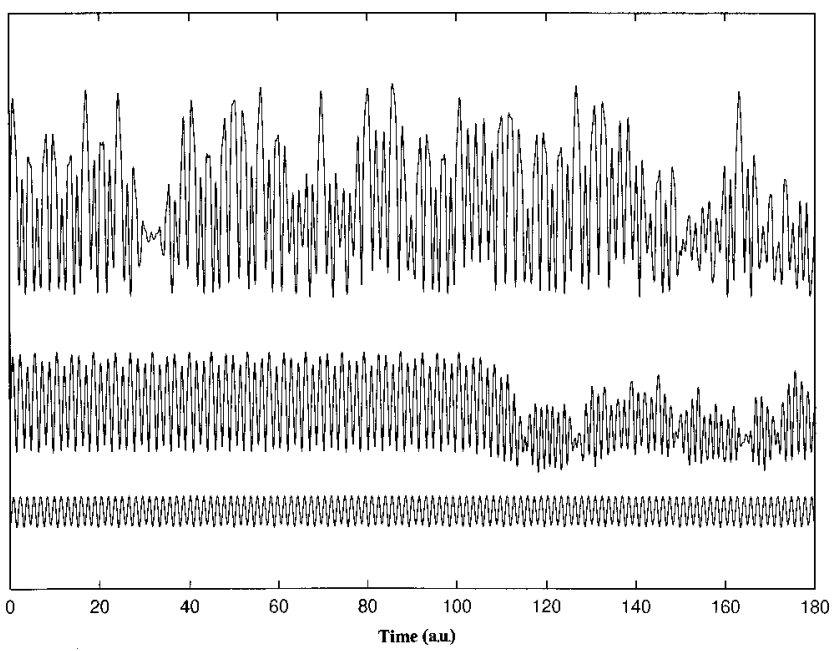

FIG. 7. The variation of the internal angle 312 .

internal angles is regular and fluctuates $2^{\circ}-3^{\circ}$ around the average value of $60^{\circ}$. The second trajectory has a relatively larger $\lambda_{\max }$ of 1.43 and we note that periodic behavior is observed during the first half of the integration. During this time the cluster displays regular but higher-amplitude vibrational motion than the first one. The internal angle fluctuations are on the order of $5^{\circ}-6^{\circ}$. Around 100 ps finally the motion becomes fully chaotic. The last trajectory is chaotic even for very short time scales, internal angles fluctuate to a great deal, and the kinetic energy is larger than in the other cases. In all trajectories the variation of the angular velocity shows the same trend from regular to chaotic motion. For the regular case the Fourier spectrum has a very sharp single peak even though there are slight irregularities in the time series. As the system becomes more chaotic, the angular velocity also displays a great deal of irregularity; however, it is important to note that the amplitudes in all cases remain reasonably unaffected by the extent of chaos, indicating that the coupling between the rotational and vibrational kineticenergy terms do not play a significant role in the extent of chaos.

\section{CONCLUSION}

We conclude that the chaotic motion of the small clusters can be analyzed in terms of energy transfer between the potential-, rotational kinetic-, and vibrational kinetic-energy terms. A series of trajectories where the initial partitioning of the kinetic energy between rotational and vibrational motion smoothly varies shows that this partitioning is the major factor determining the extent of chaos. When the initial kinetic energy is stored in the vibrational motion, it can flow from kinetic to potential energy rather freely and results in chaotic motion. On the other hand, large rotational kinetic energy hinders such flow of energy and the motion is highly regular.
[1] R. S. Berry, J. Phys. Chem. 98, 6910 (1994).

[2] D. J. Wales, Science 271, 925 (1996).

[3] D. J. Wales, J. Chem. Phys. 91, 7002 (1989).

[4] C. J. Tsai, K. D. Jordan, C. Amitrano, and R. S. Berry, J. Phys. Chem. 97, 11227 (1993).
[5] R. E. Kunz and R. S. Berry, J. Chem. Phys. 103, 1904 (1995).

[6] R. S. Berry, Chem. Rev. 93, 2379 (1993).

[7] C. Amitrano and R. S. Berry, Phys. Rev. Lett. 68, 729 (1992).

[8] R. J. Hinde and R. S. Berry, J. Chem. Phys. 99, 2942 (1993). 
[9] R. J. Hinde and R. S. Berry, Z. Phys. D 26, 391 (1993).

[10] C. Chakravarty, R. J. Hinde, D. M. Leitner, and D. J. Wales, Phys. Rev. E 56, 363 (1997).

[11] E. Yurtsever and N. Elmaci, Phys. Rev. A 55, 538 (1997).
[12] E. Yurtsever, Europhys. Lett. 37, 91 (1997).

[13] A. Wolf, J. B. Swift, H. L. Swinney, and J. A. Vastano, Physica D 16, 285 (1985).

[14] J. Jellinek and D. H. Li, Phys. Rev. Lett. 62, 241 (1989). 